\title{
On the Consistency of the Arithmetic System
}

\author{
T. J. Stępień, Ł. T. Stępień \\ The Pedagogical University of Cracow, ul. Podchorazych 2, 30 - 084 Krakow, Poland
}

Received: October 23, 2016 / Accepted: November 20, 2016 / Published: February 25, 2017.

\begin{abstract}
In this paper we establish that the well-known Arithmetic System is consistent in the traditional sense. The proof is done within this Arithmetic System.
\end{abstract}

Key words: consistency in the traditional sense, consistency in the absolute sense, Arithmetic System, Peano's Arithmetic System

\section{Introduction and Overview}

The problem of consistency of the Arithmetic System, is the well-known problem (see [1], [3], [4], [5], [7], [8], [9],[12], [14], [15], [16], [17], [19], [20], [22], [23], [24], [25], [26], [27], [28], [29], [30], [31], [32], [33], [34], [35], [37], [38], [39], [41], [42], [43], [46], [47], [48], [49], [50], [51], [52], [55], [56], [57], [58], [59], [60], [61], [63], [64], [65], [66], [67], [68], [69], [70], [71], [73], [74], [75], [76], [78], [79], [80]). In this paper we give the elementary proof of the consistency of the Arithmetic System. This result was included in the talk, presented under the similar title, at "Logic Colloquium 2009" in Sofia (Bulgaria), [59]. The abstract of this talk has been published in "The Bulletin of Symbolic Logic", [60].

\section{Terminology}

Let: $\rightarrow, \sim, \vee, \wedge, \equiv$ denote the connectives of implication, negation, disjunction, conjunction and equivalence, respectively. $\mathcal{N}=\{1,2, \ldots\}$ denotes the set of all natural numbers.

Next $\quad A t_{0}=\left\{p_{1}^{1}, p_{2}^{1}, \ldots, p_{1}^{2}, p_{2}^{2}, \ldots, p_{1}^{k}, p_{2}^{k}, \ldots\right\}(k \in$ $\mathcal{N})$ denotes the set of all propositional variables. Hence, $S_{0}$ is the set of all well-formed formulas, which are built in the usual manner from propositional

Corresponding author: Ł. T. Stępień, The Pedagogical University of Cracow, Kraków, Poland. E-mail: sfstepie@cyf-kr.edu.pl, http://www.ltstepien.up.krakow.pl variables and by means of logical connectives. $P_{0}(\phi)$ denotes the set of all propositional variables occuring in $\phi\left(\phi \in S_{0}\right) . R_{S_{0}}$ denotes the set of all rules over $S_{0}$ (see [44]). $E(\mathfrak{M})$ is the set of all formulas valid in the matrix $\mathfrak{M}$. The $\mathfrak{M}_{2}$ denotes the classical two-valued matrix. $Z_{2}$ is the set of all formulas valid in the matrix $\mathfrak{M}_{2}$ (see [44]).

The symbols $x_{1}, x_{2}, \ldots$ are individual variables. $a_{1}, a_{2}, \ldots$ are individual constants. $V$ is the set of all individual variables. $C$ is the set of all individual constants. $P_{i}^{n}(i, n \in \mathcal{N}=\{1,2, \ldots\})$ are $n$-ary predicate letters. The symbols $f_{i}^{n}(i, n \in \mathcal{N})$ are $n$-ary function letters. The symbols $\wedge x_{k}, \vee x_{k}$ are quantifiers. $\wedge x_{k}$ is the universal quantifier and $\vee x_{k}$ is the existential quantifier. The function letters, applied to the individual variables and individual constants, generate terms. The symbols $t_{1}, t_{2}, \ldots$ are terms. $T$ is the set of all terms. $V \cup C \subseteq T$. The predicate letters, applied to terms, yield simple formulas, i.e. if $P_{i}^{k}$ is a predicate letter and $t_{1}, \ldots, t_{k}$ are terms, then $P_{i}^{k}\left(t_{1}, \ldots, t_{k}\right)$ is a simple formula. Smp is the set of all simple formulas. Next, $A t_{1}$ is the set of all atomic formulas, i.e. $A t_{1}=\left\{P_{i}^{k}\left(x_{j_{1}}, \ldots, x_{j_{k}}\right)\right.$ : $\left.i, k, j_{1}, \ldots, j_{k} \in \mathcal{N}\right\}$. At last, $S_{1}$ is the set of all well-formed formulas. $F V(\phi)$ denotes the set of all free variables occuring in $\phi$, where $\phi \in S_{1}$.

$x_{k} \in F f\left(t_{m}, \phi\right)$ expresses that $x_{k}$ is free for term $t_{m}$ in $\phi\left(\phi \in S_{1}\right)$. By $x_{k} / t_{m}$ we denote the substitution of the term $t_{m}$ for the individual variable $x_{k}$. 
$P_{1}(\phi)$ denotes the set of all predicate letters occuring in $\phi\left(\phi \in S_{1}\right)$. If $F V(\phi)=\left\{x_{1}, \ldots, x_{k}\right\}$, then $\wedge \phi=\wedge x_{1} \ldots \wedge x_{k} \phi . R_{S_{1}}$ denotes the set of all rules over $S_{1} \cdot \bar{S}_{1}=\left\{\phi \in S_{1}: F V(\phi)=\emptyset\right\}$.

We use $\Rightarrow, \neg, \mathbb{V}, \&, \Leftrightarrow, \forall, \exists$ as metalogical symbols. Next, $r_{0}^{i}(i \in\{0,1\})$ denotes Modus Ponens for propositional and predicate calculi, respectively.

$r_{+}$denotes the generalization rule. $R_{0+}=\left\{r_{0}^{1}, r_{+}\right\}$.

$L_{2}$ is the set of all formulas valid in the classical calculus of quantifiers (see [45]). We write $X \subset Y$ for $X \subseteq Y$ and $Y \neq X$. For any $X \subseteq S_{i}, \operatorname{Cn}(R, X)$ is the smallest subset of $S_{i}$, containing $X$ and closed under the rules $R \subseteq R_{S_{i}}$ and $i \in\{0,1\}$. The couple $\langle R, X\rangle$ is called a system, whenever $R \subseteq R_{S_{i}}, X \subseteq S_{i}$ and $i \in\{0,1\}$ (see [44], [45], cf. [11]).

Now we repeat some well-known properties of operation of consequence and some well-known definitions (see [34], [44], [45]). Let $R \subseteq R_{S_{i}}$ and $X \subseteq S_{i}$. Then:

$$
\begin{aligned}
& \left(a_{1}\right) X \subseteq \operatorname{Cn}(R, X), \\
& \left(a_{2}\right) X \subseteq Y \Rightarrow \operatorname{Cn}(R, X) \subseteq \operatorname{Cn}(R, Y), \\
& \left(a_{3}\right) R \subseteq R^{\prime} \Rightarrow \operatorname{Cn}(R, X) \subseteq \operatorname{Cn}\left(R^{\prime}, X\right), \\
& \left(a_{4}\right) \operatorname{Cn}(R, \operatorname{Cn}(R, X)) \subseteq \operatorname{Cn}(R, X), \\
& \left(a_{5}\right) \operatorname{Cn}(R, X)=\bigcup\{\operatorname{Cn}(R, Y): Y \in \operatorname{Fin}(X)\},
\end{aligned}
$$

where $Y \in \operatorname{Fin}(X)$ denotes that $Y$ is the finite subset of $X$ and $i \in\{0,1\}$.

Definition 1.1. $\langle R, X\rangle \in C n s^{T} \Leftrightarrow\left(\neg \exists \alpha \in S_{i}\right)[\alpha \in$ $\operatorname{Cn}(R, X) \& \sim \alpha \in C n(R, X)]$, where $i \in\{0,1\}$.

Definition 1.2. $\langle R, X\rangle \in C n s^{A} \Leftrightarrow \operatorname{Cn}(R, X) \neq S_{i}$, where $i \in\{0,1\}$.

$\langle R, X\rangle \in \mathrm{Cns}^{T}$ denotes that the system $\langle R, X\rangle$ is consistent in the traditional sense, and $\langle R, X\rangle \in C n s^{A}$ denotes that the system $\langle R, X\rangle$ is consistent in the absolute sense (see [44], [45]).

\section{Basic Theorems}

Theorem 2.1. $\left\langle R_{0+}, L_{2}\right\rangle \in C n s^{T}$.

Theorem 2.2. $\left\langle R_{0+}, L_{2}\right\rangle \in C n s^{A}$.

Theorem 2.3. $\left(\forall \alpha \in \bar{S}_{1}\right)\left(\forall \beta \in S_{1}\right)\left(\forall X \subseteq S_{1}\right)$ $\left[\beta \in C n\left(R_{0+}, A_{2} \cup X \cup\{\alpha\}\right) \Rightarrow(\alpha \rightarrow \beta) \in\right.$ $\left.\operatorname{Cn}\left(R_{o+}, A_{2} \cup X\right)\right]$.
Theorem 2.4. $\left(\forall \alpha \in \bar{S}_{1}\right)\left(\forall X \subseteq S_{1}\right)$

$\left[C n\left(R_{0+}, A_{2} \cup X \cup\{\alpha\}\right)=S_{1} \Leftrightarrow \sim \alpha \in\right.$ $\left.\operatorname{Cn}\left(R_{0+}, A_{2} \cup X\right)\right]$.

Theorem 2.5. $\left(\forall \alpha \in \bar{S}_{1}\right)\left(\forall X \subseteq S_{1}\right)$

$\left[\alpha \notin \operatorname{Cn}\left(R_{0+}, A_{2} \cup X\right) \Leftrightarrow\right.$

$\left.C n\left(R_{0+}, A_{2} \cup X \cup\{\sim \alpha\}\right) \neq S_{1}\right]$,

where $A_{2}$ denotes the set of the axioms of the classical calculus of quantifiers (see [45]).

\section{Arithmetic Terminology}

Next, $S_{A}$ denotes the set of all well-formed formulas of the Arithmetic System. Hence, $F v(\phi)$ denotes the set of all free variables occuring in $\phi$, where $\phi \in S_{A}$. $x_{k} \in F f_{A}\left(t_{m}, \phi\right)$ expresses that $x_{k}$ is free for term $t_{m}$ in $\phi$, where $\phi \in S_{A}$.

$\bar{S}_{A}=\left\{\phi \in S_{A}: F v(\phi)=\emptyset\right\}$. Analogically, $R_{S_{A}}$ denotes the set of all rules over $S_{A}$. For any $X \subseteq S_{A}$ and for any $R \subseteq R_{S_{A}}, C n(R, X)$ is the smallest subset of $S_{A}$, containing $X$ and closed under the rules of $R$. The couple $\langle R, X\rangle$ is called a system, whenever $R \subseteq R_{S_{A}}$ and $X \subseteq S_{A} . R_{0+}^{P}=\left\{r_{0}^{P}, r_{+}^{P}\right\} \subseteq R_{S_{A}}$, where $r_{0}^{P}$ and $r_{+}^{P}$ are Modus Ponens and generalization rule in the Arithmetic System, respectively. By $\psi^{1}, \psi^{2}, \psi^{3}, \psi^{4}, \psi^{5}, \psi^{6}, \psi^{7}, \psi^{8}, \psi^{9}, \psi^{10}, \psi^{11}, \psi^{12}$, we denote the specific axioms of the Arithmetic System, where:

$$
\begin{aligned}
\psi^{1} \cdot & \wedge x_{1}\left(x_{1}=x_{1}\right), \\
\psi^{2} \cdot & \wedge x_{1} \wedge x_{2}\left(x_{1}=x_{2} \rightarrow x_{2}=x_{1}\right), \\
\psi^{3} . & \wedge x_{1} \wedge x_{2} \wedge x_{3}\left(x_{1}=x_{2} \rightarrow\left(x_{2}=x_{3} \rightarrow x_{1}=x_{3}\right)\right), \\
\psi^{4} . & \wedge x_{1} \wedge x_{2} \wedge x_{3} \wedge x_{4}\left(x_{1}=x_{2} \rightarrow\left(x_{3}=x_{4} \rightarrow\right.\right. \\
& \left.\left.\left(x_{1}+x_{3}=x_{2}+x_{4}\right)\right)\right) \\
\psi^{5} . & \wedge x_{1} \wedge x_{2} \wedge x_{3} \wedge x_{4}\left(x_{1}=x_{2} \rightarrow\left(x_{3}=x_{4} \rightarrow\right.\right. \\
& \left.\left.\left(x_{1} \cdot x_{3}=x_{2} \cdot x_{4}\right)\right)\right), \\
\psi^{6} . & \wedge x_{1} \wedge x_{2} \wedge x_{3} \wedge x_{4}\left(x_{1}=x_{2} \rightarrow\left(x_{3}=x_{4} \rightarrow\right.\right. \\
& \left.\left.\left(x_{1}<x_{3} \rightarrow x_{2}<x_{4}\right)\right)\right), \\
\psi^{7} \cdot & \wedge x_{1} \sim\left(1=x_{1}+1\right), \\
\psi^{8} \cdot & \wedge x_{1} \wedge x_{2}\left(x_{1}+1=x_{2}+1 \rightarrow x_{1}=x_{2}\right), \\
\psi^{9} \cdot & \wedge x_{1} \wedge x_{2}\left(x_{1}+\left(x_{2}+1\right)=\left(x_{1}+x_{2}\right)+1\right), \\
\psi^{10} \cdot & \wedge x_{1}\left(x_{1} \cdot 1=x_{1}\right), \\
\psi^{11} \cdot & \wedge x_{1} \wedge x_{2}\left[x_{1} \cdot\left(x_{2}+1\right)=\left(x_{1} \cdot x_{2}\right)+x_{1}\right], \\
\psi^{12} \cdot & \wedge x_{1} \wedge x_{2}\left[x_{1}<x_{2} \equiv \vee x_{3}\left(x_{1}+x_{3}=x_{2}\right)\right] .
\end{aligned}
$$


Hence, $X_{P}=\left\{\psi^{1}, \psi^{2}, \psi^{3}, \psi^{4}, \psi^{5}, \psi^{6}, \psi^{7}, \psi^{8}, \psi^{9}\right.$, $\left.\psi^{10}, \psi^{11}, \psi^{12}\right\}$. Next, the induction schema is the set of the following axioms:

$$
\begin{gathered}
\psi^{13} \cdot\left(\phi(1) \wedge \wedge x_{1}\left(\phi\left(x_{1}\right) \rightarrow \phi\left(x_{1}+1\right)\right)\right) \rightarrow \\
\wedge x_{1} \phi\left(x_{1}\right),
\end{gathered}
$$

where $\phi(1), \phi(x), \phi(x+1) \in S_{A}$.

Hence, $Y_{P}$ denotes here the set of all axioms of induction. Thus, $L_{2}^{r}$ and $A_{r}$ denote the set of all logical axioms and the set of all specific axioms of the Arithmetic System, respectively, where $A_{r}=X_{P} \cup Y_{P}$ (see [16], [50]).

Hence, $\left\langle R_{0+}^{P}, L_{2}^{r} \cup A_{r}\right\rangle$ is the Arithmetic System. In [50], one can read that the system $\left\langle R_{0+}^{P}, L_{2}^{r} \cup A_{r}\right\rangle$ is a modification of Peano's Arithmetic System.

$S x$ denotes here the successor of $x$ (see [34]).

Next, by $Q^{1}, Q^{2}, Q^{3}, Q^{4}, Q^{5}, Q^{6}, Q^{7}, Q^{8}, Q^{9}$, we denote the other specific axioms of the arithmetic system, where:

$$
\begin{aligned}
& Q^{1} \cdot \wedge x(x+0=x), \\
& Q^{2} \cdot \wedge x \wedge y(x \cdot S y=x \cdot y+x), \\
& Q^{3} \cdot \wedge x \wedge y(S x=S y \rightarrow x=y), \\
& Q^{4} \cdot \wedge x \vee y(y=S x), \\
& Q^{5} \cdot \wedge x \wedge y[x+S y=S(x+y)], \\
& Q^{6} \cdot \wedge x(x \cdot 0=0), \\
& Q^{7} \cdot \sim \vee x(S x+1=1), \\
& Q^{8} \cdot \wedge x_{1} \wedge x_{2}\left[\vee x_{3}\left(S x_{3}+x_{1}=x_{2}\right) \equiv\left(x_{1}<x_{2}\right)\right], \\
& Q^{9} \cdot \wedge x \sim(S x=0) . \\
& \text { Hence, } \\
& X_{P}^{\prime}=\left\{Q^{1}, Q^{2}, Q^{3}, Q^{4}, Q^{5}, Q^{6}, Q^{7}, Q^{8}, Q^{9}\right\} .
\end{aligned}
$$

Next, the induction schema is the set of the following axioms:

$$
Q^{10} .(\phi(0) \wedge \wedge x(\phi(x) \rightarrow \phi(S x))) \rightarrow \wedge x \phi(x),
$$

where $\phi(1), \phi(x), \phi(S x) \in S_{A}$.

Hence, $Y_{P}^{\prime}$ denotes the set of all axioms of induction and $A_{r}^{\prime}$ denotes the set of all specific axioms of the arithmetic system, where $A_{r}^{\prime}=X_{P}^{\prime} \cup Y_{P}^{\prime}$.

In consequence, one can obtain another arithmetic system (cf. [1], [2], [4], [6], [7], [10], [13], [14], [16],[17], [18], [21], [23], [25], [28], [30], [31], [32],
[34], [36], [37], [40], [47], [48], [50], [51], [52], [53], [54],[58], [59], [62], [71], [72], [77], [78]), namely, $\left\langle R_{o+}^{P}, L_{2}^{r^{\prime}} \cup A_{r}^{\prime}\right\rangle$, where $L_{2}^{r^{\prime}}$ is the set of all logical axioms.

$L_{1}^{2}$ denotes the well-known subset of the set $L_{2}^{r}$ (see[16], [44], [45], [50]). Namely, $L_{1}^{2}=\left\{\phi^{1}, \phi^{2}, \phi^{3}\right.$, $\left.\phi^{4}, \phi^{5}, \phi^{6}, \phi^{7}, \phi^{8}, \phi^{9}, \phi^{10}, \phi^{11}, \phi^{12}\right\}$, where:

$$
\begin{aligned}
& \phi^{1} .[\alpha \rightarrow(\beta \rightarrow \gamma)] \rightarrow[(\alpha \rightarrow \beta) \rightarrow(\alpha \rightarrow \gamma)], \\
& \phi^{2} .(\sim \alpha \rightarrow \alpha) \rightarrow \alpha, \\
& \phi^{3} . \sim \alpha \rightarrow(\alpha \rightarrow \beta), \\
& \phi^{4} . \alpha \rightarrow(\beta \rightarrow \alpha), \\
& \phi^{5} . \alpha \wedge \beta \rightarrow \alpha, \\
& \phi^{6} . \alpha \wedge \beta \rightarrow \beta, \\
& \phi^{7} . \alpha \rightarrow(\beta \rightarrow \alpha \wedge \beta), \\
& \phi^{8} . \alpha \rightarrow \alpha \vee \beta, \\
& \phi^{9} . \beta \rightarrow \alpha \vee \beta, \\
& \phi^{10} .(\alpha \rightarrow \beta) \rightarrow[(\delta \rightarrow \beta) \rightarrow(\alpha \vee \delta \rightarrow \beta)], \\
& \phi^{11} . \wedge x_{k} \phi \rightarrow \phi\left(\frac{x_{k}}{t_{n}}\right), \text { if } x_{k} \in F f_{A}\left(t_{n}, \phi\right), \\
& \phi^{12} . \wedge x_{k}(\phi \rightarrow \psi) \rightarrow\left(\phi \rightarrow \wedge x_{k} \psi\right), \text { if } x_{k} \notin F v(\phi) \\
& \text { and } \\
& \alpha, \beta, \gamma, \delta, \phi, \psi \in S_{A} .
\end{aligned}
$$

The analogons of Definition 1.1., Definition 1.2., Theorem 2.1., Theorem 2.2., Theorem 2.4. and Theorem 2.5., are the following (where $R \subseteq R_{S_{A}}$ and $\left.X \subseteq S_{A}\right)$ :

Definition 3.1. $\langle R, X\rangle \in C n s_{A}^{T} \Leftrightarrow\left(\neg \exists \alpha \in S_{A}\right)$ $[\alpha \in \operatorname{Cn}(R, X) \& \sim \alpha \in \operatorname{Cn}(R, X)]$.

Definition 3.2. $\langle R, X\rangle \in C n s_{A}^{A} \Leftrightarrow C n(R, X) \neq S_{A}$.

Theorem 3.3. $\left\langle R_{0+}^{P}, L_{2}^{r}\right\rangle \in C n s_{A}^{T}$.

Theorem 3.4. $\left\langle R_{0+}^{P}, L_{2}^{r}\right\rangle \in C n s_{A}^{A}$.

Theorem 3.5. $\left(\forall \alpha \in \bar{S}_{A}\right)\left(\forall X \subseteq S_{A}\right)$

$$
\begin{aligned}
& {\left[C n\left(R_{0+}^{P}, L_{1}^{2} \cup X \cup\{\alpha\}\right)=S_{A} \Leftrightarrow\right.} \\
& \left.\sim \alpha \in C n\left(R_{0+}^{P}, L_{1}^{2} \cup X\right)\right] .
\end{aligned}
$$

Theorem 3.6. $\left(\forall \alpha \in \bar{S}_{A}\right)\left(\forall X \subseteq S_{A}\right)$

$$
\begin{aligned}
& {\left[\alpha \notin C n\left(R_{0+}^{P}, L_{1}^{2} \cup X\right) \Leftrightarrow\right.} \\
& \left.\quad C n\left(R_{0+}^{P}, L_{1}^{2} \cup X \cup\{\sim \alpha\}\right) \neq S_{A}\right],
\end{aligned}
$$

where $L_{1}^{2} \subseteq L_{2}^{r}$ (see [11], [16], [34], [45], [50], [65]). 


\section{The Basic Corollaries and Lemmas}

At first, we introduce the following formulas:

(I $I_{1} O_{0}=\psi^{7} \equiv \sim \sim \psi^{1}$,

(I $\left.I_{2}\right) u_{27}=\sim(1<1)$,

(I $\left.I_{3}\right) O_{6}=O_{0} \rightarrow\left(\psi^{7} \rightarrow \psi^{1}\right)$,

(I $\left.I_{4}\right) \alpha_{2}^{x}=\psi^{1} \rightarrow \psi^{7}$,

(I $\left.I_{5}\right) \gamma_{2}^{\prime}=O_{0} \rightarrow u_{27}$,

(I $\left.I_{6}\right) \gamma_{0}^{\prime}=\left(\psi^{7} \rightarrow \psi^{1}\right) \rightarrow \psi^{12}$,

(I $\left.I_{7}\right) \gamma_{0}=u_{27} \rightarrow \gamma_{0}^{\prime}$,

(I $\left.I_{8}\right) \gamma_{4}^{\prime}=\gamma_{0}^{\prime} \rightarrow O_{0}$.

Next, we assume that

$\left(I_{9}\right)\left(\forall \alpha, \delta \in S_{A}\right)\left[{ }_{\alpha} \delta=\alpha \rightarrow \delta\right]$,

$\left(I_{10}\right)\left(\forall X \subseteq S_{A}\right)\left(\forall \alpha \in S_{A}\right)\left[{ }_{\alpha} X=(\alpha \rightarrow \beta: \beta \in X)\right]$.

Next, we define the sets $L_{1}^{1}$ and ${ }_{\psi^{7} O_{0} u_{27} \sim \psi^{1}} L_{2}^{r}$, as follows:

$$
\begin{gathered}
\left(I_{11}\right) L_{1}^{1}=L_{1}^{2} \cup\left\{\psi^{1} \rightarrow\left(\psi^{7} \rightarrow\left(\psi^{12} \rightarrow \omega\right)\right):\right. \\
\left.\omega \in L_{2}^{r}-L_{1}^{2}\right\}, \\
\left(I_{12}\right){ }_{\psi^{7} O_{0} u_{27} \sim \psi^{1}} L_{2}^{r}=\left\{\psi ^ { 7 } \rightarrow \left(O _ { 0 } \rightarrow \left(u_{27} \rightarrow\right.\right.\right. \\
\left.\left.\left.\left(\sim \psi^{1} \rightarrow \beta\right)\right)\right): \beta \in L_{2}^{r}-L_{1}^{2}\right\} .
\end{gathered}
$$

Lemma 4.1. $\left\langle R_{0+}^{P}, L_{2}^{r} \cup\left\{\psi^{1}, \psi^{7}, \psi^{12}\right\}\right\rangle \notin C n s_{A}^{T} \Rightarrow$ $\left(\forall \alpha \in \bar{S}_{A}-A^{0}\right)\left(\forall \delta \in \bar{S}_{A}-A^{0}\right)$

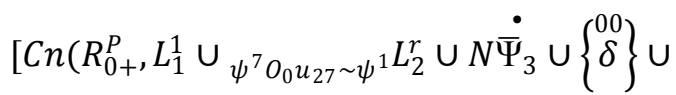

$\left.\left\{\alpha \rightarrow \psi^{7}\right\} \cup\{\xi\}\right)=S_{A} \Rightarrow \operatorname{Cn}\left(R_{0+}^{P}, L_{1}^{1} \cup\right.$

$\left.\left.\psi^{7} o_{0} u_{27} \sim \psi^{1} L_{2}^{r} \cup N \dot{\bar{\Psi}}_{3} \cup\left\{\alpha \rightarrow \psi^{7}\right\} \cup\{\xi\}\right)=S_{A}\right]$,

where

$$
\begin{aligned}
& \stackrel{00}{\delta}=\psi^{7} \rightarrow \stackrel{0}{\delta}, \xi=\psi^{7} \rightarrow\left(\psi^{1} \rightarrow \psi^{12}\right), \\
& A^{0}=C n\left(R_{0+}^{P}, L_{1}^{1} \cup{ }_{\psi^{7} O_{0} u_{27} \sim \psi^{1}} L_{2}^{r} \cup N \dot{\bar{\Psi}}_{3} \cup\{\xi\}\right), \\
& N \dot{\bar{\Psi}}_{3}=\left\{O_{0} \rightarrow \gamma_{0}, \gamma_{2}^{\prime}, \gamma_{4}^{\prime}\right\} .
\end{aligned}
$$

Proof. Let

(1) $\left\langle R_{0+}^{P}, L_{2}^{r} \cup\left\{\psi^{1}, \psi^{7}, \psi^{12}\right\}\right\rangle \notin C n s_{A}^{T}$
(2) $\neg\left(\forall \alpha \in \bar{S}_{A}-A^{0}\right)\left(\forall \delta \in \bar{S}_{A}-A^{0}\right)$

$$
\begin{aligned}
& {\left[C n \left(R_{0+}^{P}, L_{1}^{1} \cup_{\psi^{7} O_{0} u_{27} \sim \psi^{1}} L_{2}^{r} \cup N \dot{\bar{\Psi}}_{3} \cup\right.\right.} \\
& \left.\left\{\begin{array}{l}
00 \\
\delta
\end{array}\right\} \cup\left\{\alpha \rightarrow \psi^{7}, \xi\right\}\right)=S_{A} \Rightarrow \\
& C n\left(R_{0+}^{P}, L_{1}^{1} \cup_{\psi^{7} O_{0} u_{27} \sim \psi^{1}} L_{2}^{r} \cup N \dot{\bar{\Psi}}_{3} \cup\right. \\
& \left.\left.\left\{\alpha \rightarrow \psi^{7}, \xi\right\}\right)=S_{A}\right],
\end{aligned}
$$

where

(3) $\stackrel{00}{\delta}=\psi^{7} \rightarrow \stackrel{0}{\delta}$

(4) $\xi=\psi^{7} \rightarrow\left(\psi^{1} \rightarrow \psi^{12}\right)$,

(5) $A^{0}=\operatorname{Cn}\left(R_{0+}^{P}, L_{1}^{1} \cup{ }_{\psi^{7} O_{0} u_{27} \sim \psi^{1}} L_{2}^{r} \cup\right.$

$$
\left.N \dot{\bar{\Psi}}_{3} \cup\{\xi\}\right)
$$

(6) $N \dot{\bar{\Psi}}_{3}=\left\{O_{0} \rightarrow \gamma_{0}, \gamma_{2}^{\prime}, \gamma_{4}^{\prime}\right\}$.

Hence, it follows that

(7) $\left(\exists \alpha^{\prime} \in \bar{S}_{A}-A^{0}\right)\left(\exists \stackrel{00}{\delta^{\prime}} \in \bar{S}_{A}-A^{0}\right)$

$$
\left[C n \left(R_{0+}^{P}, L_{1}^{1} \cup \psi_{\psi^{7} O_{0} u_{27} \sim \psi^{1}} L_{2}^{r} \cup N \dot{\bar{\Psi}}_{3} \cup\right.\right.
$$

$$
\begin{aligned}
& \left.\left\{\begin{array}{l}
00 \\
\delta^{\prime}
\end{array}\right\} \cup\left\{\alpha^{\prime} \rightarrow \psi^{7}, \xi\right\}\right)=S_{A} \& \operatorname{Cn}\left(R_{0+}^{P}, L_{1}^{1} \cup\right. \\
& \left.\psi^{7} O_{0} u_{27} \sim \psi^{1} L_{2}^{r} \cup N \dot{\bar{\Psi}}_{3} \cup\left\{\alpha^{\prime} \rightarrow \psi^{7}, \xi\right\}\right)= \\
& \left.A \neq S_{A}\right],
\end{aligned}
$$

where

(8) $\stackrel{00}{\delta} \delta^{\prime}=\psi^{7} \rightarrow \stackrel{0}{\delta^{\prime}}$,

(9) $\xi=\psi^{7} \rightarrow\left(\psi^{1} \rightarrow \psi^{12}\right)$,

(10) $A^{0}=\operatorname{Cn}\left(R_{0+}^{P}, L_{1}^{1} \cup_{\psi^{7} O_{0} u_{27} \sim \psi^{1}} L_{2}^{r} \cup\right.$

$\left.N \dot{\bar{\Psi}}_{3} \cup\{\xi\}\right)$,

(11) $N \dot{\bar{\Psi}}_{3}=\left\{O_{0} \rightarrow \gamma_{0}, \gamma_{2}^{\prime}, \gamma_{4}^{\prime}\right\}$.

and 
From (7) - (11), it follows that

(12) $\left(\exists \alpha^{\prime} \in \bar{S}_{A}-A^{0}\right)\left(\exists{ }^{00} \delta^{\prime} \in \bar{S}_{A}-A^{0}\right)$

$\left[C n\left(R_{0+}^{P}, L_{1}^{1} \cup \psi_{\psi^{7} O_{0} u_{27} \sim \psi^{1}} L_{2}^{r} \cup N \dot{\bar{\Psi}}_{3} \cup\right.\right.$

$\left.\left.\left\{\alpha^{\prime} \rightarrow \psi^{7}, \xi\right\}\right)=A \& A \neq S_{A} \& \stackrel{00}{\delta}^{\prime} \notin A\right]$.

From (12), by Theorem 3.6., one can obtain that

(13) $\left(\exists \alpha^{\prime} \in \bar{S}_{A}-A^{0}\right)\left(\exists \delta^{\prime} \in \bar{S}_{A}-A^{0}\right)$

$\left[C n\left(R_{0+}^{P}, L_{1}^{1} \cup{ }_{\psi^{7} O_{0} u_{27} \sim \psi^{1}} L_{2}^{r} \cup N \dot{\bar{\Psi}}_{3} \cup\right.\right.$

$\left.\left\{\alpha^{\prime} \rightarrow \psi^{7}, \xi\right\}\right)=A \& \operatorname{Cn}\left(R_{0+}^{P}, L_{1}^{1} \cup\right.$

$\psi^{7} O_{0} u_{27} \sim \psi^{1} L_{2}^{r} \cup N \dot{\bar{\Psi}}_{3} \cup\left\{\sim \delta^{\prime \prime}\right\} \cup$

$\left.\left.\left\{\alpha^{\prime} \rightarrow \psi^{7}\right\} \cup\{\xi\}\right)=A^{*} \& A^{*} \neq S_{A}\right]$.

Hence, from (1), (8), (9), (11), $\left(I_{5}\right),\left(I_{6}\right),\left(I_{8}\right)$, $\left(I_{11}\right),\left(I_{12}\right)$ and Theorem 3.5., it follows that

(14) $\left(\exists \alpha^{\prime} \in \bar{S}_{A}-A^{0}\right)\left(\exists \delta^{00} \in \bar{S}_{A}-A^{0}\right)$

$\left[C n\left(R_{0+}^{P}, L_{1}^{1} \cup_{\psi^{7} O_{0} u_{27} \sim \psi^{1}} L_{2}^{r} \cup N \dot{\bar{\Psi}}_{3} \cup\right.\right.$

$\left.\left\{\sim^{00} \delta^{\prime}\right\} \cup\left\{\alpha^{\prime} \rightarrow \psi^{7}\right\} \cup\{\xi\}\right)=A^{*} \&$

$\psi^{7}, \gamma_{4}^{\prime}, \gamma_{2}^{\prime}, O_{0} \rightarrow \gamma_{0}, \psi^{1} \rightarrow \psi^{12}, \gamma_{0}^{\prime}$,

$O_{0}, u_{27}, \psi^{12} \rightarrow\left(\psi^{7} \rightarrow \sim \psi^{1}\right), \psi^{12} \rightarrow \sim \psi^{1}$,

$\left.\sim \psi^{1} \in A^{*} \& A^{*} \neq S_{A}\right]$.

Hence and from $\left(I_{12}\right)$, it follows that

(15) $\left(\exists \alpha^{\prime} \in \bar{S}_{A}-A^{0}\right)\left(\exists \delta^{\prime 0} \in \bar{S}_{A}-A^{0}\right)$

$\left[C n\left(R_{0+}^{P}, L_{1}^{1} \cup \psi_{\psi^{7} O_{0} u_{27} \sim \psi^{1}} L_{2}^{r} \cup N \dot{\Psi}_{3} \cup\right.\right.$

$\left.\left\{\sim^{00} \delta^{\prime}\right\} \cup\left\{\alpha^{\prime} \rightarrow \psi^{7}\right\} \cup\{\xi\}\right)=A^{*} \&$

$\left.L_{2}^{r} \subseteq A^{*} \& A^{*} \neq S_{A}\right]$.

From (14), (15) and $\left(I_{1}\right)$, it follows that

(16) $\left(\exists \alpha^{\prime} \in \bar{S}_{A}-A^{0}\right)\left(\exists \delta^{\prime 0} \in \bar{S}_{A}-A^{0}\right)$

$\left[C n\left(R_{0+}^{P}, L_{1}^{1} \cup \psi_{\psi^{7} O_{0} u_{27} \sim \psi^{1}} L_{2}^{r} \cup N \dot{\bar{\Psi}}_{3} \cup\right.\right.$

$\left.\left\{\sim^{00} \delta^{\prime}\right\} \cup\left\{\alpha^{\prime} \rightarrow \psi^{7}\right\} \cup\{\xi\}\right)=A^{*} \&$ $\psi^{7}, \psi^{7} \rightarrow \psi^{1}, \psi^{1}, \sim \psi^{1} \in A^{*} \&$

$\left.A^{*} \neq S_{A} \& A^{*}=S_{A}\right]$.

Hence, it follows that

(17) $\left(\exists \alpha^{\prime} \in \bar{S}_{A}-A^{0}\right)\left(\exists^{00} \delta^{\prime} \in \bar{S}_{A}-A^{0}\right)$

$\left[C n\left(R_{0+}^{P}, L_{1}^{1} \cup{ }_{\psi^{7} O_{0} u_{27} \sim \psi^{1}} L_{2}^{r} \cup N \dot{\bar{\Psi}}_{3} \cup\right.\right.$

$\left.\left\{\sim^{00} \delta^{\prime}\right\} \cup\left\{\alpha^{\prime} \rightarrow \psi^{7}\right\} \cup\{\xi\}\right)=A^{*} \&$

$\left.A^{*} \neq S_{A} \& A^{*}=S_{A}\right]$.

Contradiction

Lemma 4.2. $\left\langle R_{0+}^{P}, L_{2}^{r} \cup\left\{\psi^{1}, \psi^{7}, \psi^{12}\right\}\right\rangle \notin C n s_{A}^{T} \Rightarrow$

$\left(\exists \alpha^{\prime} \in \bar{S}_{A}-A^{0}\right)\left(\forall \delta \in \bar{S}_{A}-A^{0}\right)\left[\operatorname{Cn}\left(R_{0+}^{P}, L_{1}^{1} \cup\right.\right.$

$\left.\left.\psi^{7} o_{0} u_{27} \sim \psi^{1} L_{2}^{r} \cup N \dot{\bar{\Psi}}_{3} \cup\left\{\begin{array}{l}00 \\ \delta\end{array}\right\}\left\{\alpha^{\prime} \rightarrow \psi^{7}, \xi\right\}\right)=S_{A}\right]$,

where

$\stackrel{00}{\delta}=\psi^{7} \rightarrow \stackrel{0}{\delta}, \xi=\psi^{7} \rightarrow\left(\psi^{1} \rightarrow \psi^{12}\right)$,

$A^{0}=C n\left(R_{0+}^{P}, L_{1}^{1} \cup \psi_{\psi^{7} O_{0} u_{27} \sim \psi^{1}} L_{2}^{r} \cup N \dot{\bar{\Psi}}_{3} \cup\{\xi\}\right)$,

$N \dot{\Psi}_{3}=\left\{O_{0} \rightarrow \gamma_{0}, \gamma_{2}^{\prime}, \gamma_{4}^{\prime}\right\}$.

Proof. Suppose to the contrary that

(1) $\left\langle R_{0+}^{P}, L_{2}^{r} \cup\left\{\psi^{1}, \psi^{7}, \psi^{12}\right\}\right\rangle \notin C n s_{A}^{T}$

and

(2) $\neg\left(\exists \alpha^{\prime} \in \bar{S}_{A}-A^{0}\right)\left(\forall \delta \in \bar{S}_{A}-A^{0}\right)$

$$
\left[C n \left(R_{0+}^{P}, L_{1}^{1} \cup \psi_{\psi^{7} O_{0} u_{27} \sim \psi^{1}} L_{2}^{r} \cup N \dot{\bar{\Psi}}_{3} \cup\left\{\begin{array}{l}
00 \\
\delta
\end{array}\right\} \cup\right.\right.
$$$$
\left.\left.\left\{\alpha^{\prime} \rightarrow \psi^{7}, \xi\right\}\right)=S_{A}\right],
$$

where

(3) $\stackrel{00}{\delta}=\psi^{7} \rightarrow \stackrel{0}{\delta}$,

(4) $\xi=\psi^{7} \rightarrow\left(\psi^{1} \rightarrow \psi^{12}\right)$,

(5) $A^{0}=\operatorname{Cn}\left(R_{0+}^{P}, L_{1}^{1} \cup{ }_{\psi^{7} O_{0} u_{27} \sim \psi^{1}} L_{2}^{r} \cup\right.$

$$
\left.N \dot{\bar{\Psi}}_{3} \cup\{\xi\}\right)
$$

(6) $N \dot{\bar{\Psi}}_{3}=\left\{O_{0} \rightarrow \gamma_{0}, \gamma_{2}^{\prime}, \gamma_{4}^{\prime}\right\}$. 
From (2) - (6), it follows that

(7) $\left(\forall \alpha \in \bar{S}_{A}-A^{0}\right)\left(\exists{ }^{00} \delta^{\prime} \in \bar{S}_{A}-A^{0}\right)$

$$
\begin{aligned}
& {\left[C n \left(R_{0+}^{P}, L_{1}^{1} \cup{ }_{\psi^{7} O_{0} u_{27} \sim \psi^{1}} L_{2}^{r} \cup N \dot{\bar{\Psi}}_{3} \cup\left\{\delta^{\prime}\right\} \cup\right.\right.} \\
& \left.\left.\left\{\alpha \rightarrow \psi^{7}, \xi\right\}\right)=A^{+} \neq S_{A}\right],
\end{aligned}
$$

where

(8) $\stackrel{00}{\delta}{ }^{\prime}=\psi^{7} \rightarrow \stackrel{0}{\delta^{\prime}}$,

(9) $\xi=\psi^{7} \rightarrow\left(\psi^{1} \rightarrow \psi^{12}\right)$,

(10) $A^{0}=\operatorname{Cn}\left(R_{0+}^{P}, L_{1}^{1} \cup_{\psi^{7} O_{0} u_{27} \sim \psi^{1}} L_{2}^{r} \cup\right.$

$\left.N \dot{\bar{\Psi}}_{3} \cup\{\xi\}\right)$

(11) $N \dot{\bar{\Psi}}_{3}=\left\{O_{0} \rightarrow \gamma_{0}, \gamma_{2}^{\prime}, \gamma_{4}^{\prime}\right\}$

From (10) and $\left(I_{11}\right)$, it follows that

(12) $\sim \psi^{7} \in A^{0} \Rightarrow\left(\forall{ }^{0} \in \bar{S}_{A}\right)\left[\psi^{7} \rightarrow{ }^{0} \delta \in A^{0}\right]$.

Hence, from (8), it follows that

(13) $\sim \psi^{7} \in A^{0} \Rightarrow \stackrel{00}{\delta^{\prime}} \in A^{0}$.

Hence, from (7) - (11), it follows that

(14) $\sim \psi^{7} \notin A^{0}$.

From (1), (7) - (11), $\left(I_{5}\right),\left(I_{6}\right),\left(I_{11}\right),\left(I_{12}\right)$

and Theorem 3.5., it follows that

(15) $\left(\forall \alpha \in \bar{S}_{A}-A^{0}\right)\left(\exists{ }^{00} \delta^{\prime} \in \bar{S}_{A}-A^{0}\right)$

$\left[C n\left(R_{0+}^{P}, L_{1}^{1} \cup{ }_{\psi^{7} O_{0} u_{27} \sim \psi^{1}} L_{2}^{r} \cup N \dot{\bar{\Psi}}_{3} \cup\left\{\begin{array}{l}00 \\ \delta^{\prime}\end{array}\right\} \cup\right.\right.$

$\left.\left\{\alpha \rightarrow \psi^{7}, \xi\right\}\right)=A^{+} \& A^{+} \neq S_{A} \&$

$O_{0} \rightarrow \gamma_{0}, \gamma_{2}^{\prime}, \gamma_{4}^{\prime}, \psi^{1} \rightarrow\left(\psi^{7} \rightarrow \psi^{12}\right)$,

$\psi^{12} \rightarrow\left(\psi^{7} \rightarrow \sim \psi^{1}\right), \psi^{7} \rightarrow \sim \psi^{1}, \gamma_{0}^{\prime} \rightarrow O_{0}$,

$\psi^{7} \rightarrow\left(\sim \psi^{1} \rightarrow O_{0}\right), \psi^{7} \rightarrow O_{0}, O_{0} \rightarrow \gamma_{0}^{\prime}$,

$\left.\psi^{7} \rightarrow \gamma_{0}^{\prime}, \psi^{7} \rightarrow u_{27} \in A^{+}\right]$.

From (15), $\left(I_{5}\right)$, it follows that

(16) $\left(\forall \alpha \in \bar{S}_{A}-A^{0}\right)\left(\exists{ }^{00} \delta^{\prime} \in \bar{S}_{A}-A^{0}\right)$

$$
\left[\psi^{7} O_{0} \sim \psi^{1} L_{2}^{r} \subseteq A^{+}\right] .
$$

Hence, from (15), it follows that
(17) $\left(\forall \alpha \in \bar{S}_{A}-A^{0}\right)\left(\exists{ }^{00} \delta^{\prime} \in \bar{S}_{A}-A^{0}\right)\left[O_{6} \in A^{+}\right]$, where

(18) $O_{6}=O_{0} \rightarrow\left(\psi^{7} \rightarrow \psi^{1}\right)$.

Hence, from (15), $\left(I_{11}\right)$, it follows that

(19) $\left(\forall \alpha \in \bar{S}_{A}-A^{0}\right)\left(\exists \delta^{00}, \in \bar{S}_{A}-A^{0}\right)$

$$
\begin{aligned}
& {\left[\operatorname { C n } \left(R_{0+}^{P}, L_{1}^{1} \cup{ }_{\psi^{7} O_{0} u_{27} \sim \psi^{1}} L_{2}^{r} \cup N \dot{\bar{\Psi}}_{3} \cup\right.\right.} \\
& \left.\left\{\delta^{\prime}\right\} \cup\left\{\alpha \rightarrow \psi^{7}, \xi\right\}\right)=A^{+} \& A^{+} \neq S_{A} \& \\
& \gamma_{0}^{\prime} \rightarrow\left(\psi^{7} \rightarrow \psi^{1}\right), \psi^{7} \rightarrow \psi^{1}, \psi^{1} \rightarrow \sim \psi^{7}, \\
& \left.\sim \psi^{7} \in A^{+}\right] .
\end{aligned}
$$

Hence, from (10), it follows that

(20) $\left(\forall \alpha \in \bar{S}_{A}-A^{0}\right)\left(\exists^{00} \delta^{\prime} \in \bar{S}_{A}-A^{0}\right)$

$$
\begin{aligned}
& {\left[C n \left(R_{0+}^{P}, L_{1}^{1} \cup{ }_{\psi^{7} O_{0} u_{27} \sim \psi^{1}} L_{2}^{r} \cup N \dot{\bar{\Psi}}_{3} \cup\left\{\delta^{00}\right\} \cup\right.\right.} \\
& \left.\left\{\alpha \rightarrow \psi^{7}, \xi\right\}\right)=A^{+} \& A^{+} \neq S_{A} \& \\
& \left.A^{+}-A^{0}=\emptyset \& A^{0} \subseteq A^{+}\right] .
\end{aligned}
$$

Hence, from (10) and (19), it follows that

(21) $\sim \psi^{7} \in A^{0}$,

what contradicts with (14).

Theorem 4.1. $\left\langle R_{0+}^{P}, L_{2}^{r} \cup\left\{\psi^{1}, \psi^{7}, \psi^{12}\right\}\right\rangle \in C n s_{A}^{T}$.

Proof. Let

(1) $\left\langle R_{0+}^{P}, L_{2}^{r} \cup\left\{\psi^{1}, \psi^{7}, \psi^{12}\right\}\right\rangle \notin C n s_{A}^{T}$.

From (1), by Lemma 4.1. and Lemma 4.2., it follows that

(2) $\left(\exists \alpha^{\prime} \in \bar{S}_{A}-A^{0}\right)\left[\operatorname{Cn}\left(R_{0+}^{P}, L_{1}^{1} \cup{ }_{\psi^{7} O_{0} u_{27} \sim \psi^{1}} L_{2}^{r} \cup\right.\right.$

$$
\left.\left.N \dot{\bar{\Psi}}_{3} \cup\left\{\alpha^{\prime} \rightarrow \psi^{7}\right\} \cup\{\xi\}\right)=S_{A}\right],
$$

where

(3) $A^{0}=\operatorname{Cn}\left(R_{0+}^{P}, L_{1}^{1} \cup{ }_{\psi^{7} O_{0} u_{27} \sim \psi^{1}} L_{2}^{r} \cup\right.$

$$
\left.N \dot{\bar{\Psi}}_{3} \cup\{\xi\}\right) \text {. }
$$

Hence, by Theorem 3.5., it follows that

(4) $\left(\exists \alpha^{\prime} \in \bar{S}_{A}-A^{0}\right)\left[\sim\left(\alpha^{\prime} \rightarrow \psi^{7}\right) \in A^{0}\right]$,

where 
(5) $A^{0}=C n\left(R_{0+}^{P}, L_{1}^{1} \cup{ }_{\psi^{7} O_{0} u_{27} \sim \psi^{1}} L_{2}^{r} \cup N \dot{\bar{\Psi}}_{3} \cup\right.$ $\{\xi\})$.

Hence, it follows that

(6) $\left(\exists \alpha^{\prime} \in \bar{S}_{A}\right)\left[\alpha^{\prime} \notin A^{0} \& \alpha^{\prime} \in A^{0}\right]$,

where

(7) $A^{0}=\operatorname{Cn}\left(R_{0+}^{P}, L_{1}^{1} \cup_{\psi^{7} O_{0} u_{27} \sim \psi^{1}} L_{2}^{r} \cup\right.$

$$
\left.N \dot{\bar{\Psi}}_{3} \cup\{\xi\}\right) .
$$

Contradiction

Now,

Corollary 4.1. $\left\langle R_{0+}^{P}, L_{2}^{r} \cup A_{r}\right\rangle \notin C n s_{A}^{T} \Rightarrow$ $\left(\exists Y_{P}^{\prime \prime} \subseteq Y_{P}\right)\left(\exists X_{p}^{\prime \prime} \subseteq X_{P}\right)\left\{C n\left(R_{0+}^{P}, L_{2}^{r} \cup Y_{p}^{\prime \prime} \cup X_{P}^{\prime \prime}\right)=\right.$ $\left.S_{A} \&\left(\forall Y \subset X_{P}^{\prime \prime} \cup Y_{P}^{\prime \prime}\right)\left[C n\left(R_{0+}^{P}, L_{2}^{r} \cup Y\right) \neq S_{A}\right]\right\}$, where

$Y_{P}^{\prime \prime}=\left\{\alpha_{1}, \ldots, \alpha_{k}\right\}, X_{p}^{\prime \prime}=\left\{\alpha_{1}^{\prime}, \ldots, \alpha_{n}^{\prime}\right\}$ and $k, n \in \mathcal{N}$ and $X_{P} \cup Y_{P}=A_{r}$.

Proof. By Theorem 3.3. - Theorem 3.6., by the definition of operation of consequence, and by the definitions of the sets $L_{2}^{r}$ and $A_{r}$.

Corollary 4.2. $\left\langle R_{0+}^{P}, L_{2}^{r} \cup A_{r}\right\rangle \notin C n s_{A}^{T} \Rightarrow\left(\exists Y_{P}^{\prime \prime} \subseteq Y_{P}\right)$ $\left(\exists X_{p}^{\prime \prime} \subseteq X_{P}\right)\left[C n\left(R_{0+}^{P}, L_{2}^{r} \cup Y_{P}^{\prime \prime} \cup X_{P}^{\prime \prime}\right)=S_{A} \&\right.$ $\left(\forall Y \subset X_{P}^{\prime \prime} \cup Y_{P}^{\prime \prime}\right)\left[C n\left(R_{0+}^{P}, L_{2}^{r} \cup Y\right) \neq S_{A} \&\right.$ $\left(\exists Z_{P} \subseteq\left\{\psi^{2}, \psi^{3}, \psi^{4}, \psi^{5}, \psi^{6}, \psi^{8}, \psi^{9}, \psi^{10}, \psi^{11}\right\} \cup Y_{P}\right)$ $\left.\left[Z_{P} \subseteq X_{P}^{\prime \prime} \cup Y_{P}^{\prime \prime} \& Z_{P} \neq \varnothing\right]\right]$,

where

$Y_{P}^{\prime \prime}=\left\{\alpha_{1}, \ldots, \alpha_{k}\right\}, X_{P}^{\prime \prime}=\left\{\alpha_{1}^{\prime}, \ldots, \alpha_{n}^{\prime}\right\}$ and $k, n \in \mathcal{N}$ and $Y_{P} \cup X_{P}=A_{r}$.

Proof. By Corollary 4.1. and by Theorem 4.1.

Using Corollary 4.2., we define the formulas $\stackrel{0}{\beta}, \stackrel{1}{\beta}$, as follows:

$$
\begin{aligned}
\left(I_{14}\right) \stackrel{0}{\beta}= & \alpha_{1} \wedge \ldots \wedge \alpha_{k} \wedge \alpha_{1}^{\prime} \wedge \ldots \wedge \alpha_{n}^{\prime} \wedge \psi^{1} \wedge \psi^{7} \wedge \\
& \psi^{12}, \\
\left(I_{15}\right) \stackrel{1}{\beta}= & \psi^{1} \rightarrow\left(\psi^{7} \rightarrow\left(\psi^{12} \rightarrow \stackrel{0}{\beta}\right)\right),
\end{aligned}
$$

where

$\left\{\alpha_{1}, \ldots, \alpha_{k}\right\}=Y_{P}^{\prime \prime}$ and $\left\{\alpha_{1}^{\prime}, \ldots, \alpha_{n}^{\prime}\right\}=X_{P}^{\prime \prime}$ and $k, n \in \mathcal{N}$.
Next, we define some sets $L_{T}^{1}$ and $N \ddot{\bar{\Psi}}_{3}$, as follows:

$\left(I_{16}\right) L_{T}^{1}=L_{1}^{2} \cup\left\{\begin{array}{l}0 \\ \beta\end{array} \rightarrow \omega: \omega \in L_{2}^{r}-L_{1}^{2}\right\}$,

$\left(I_{17}\right) N \ddot{\bar{\Psi}}_{3}=\left\{O_{0} \rightarrow\left(u_{27} \rightarrow \stackrel{1}{\beta}\right), O_{0} \rightarrow \gamma_{0}, \gamma_{2}^{\prime}, \gamma_{4}^{\prime}\right\}$.

Thus,

Corollary 4.3. $\left\langle R_{0+}^{P}, L_{2}^{r} \cup A_{r}\right\rangle \notin C n s_{A}^{T} \Rightarrow$ $C n\left(R_{0+}^{P}, L_{T}^{1} \cup\left\{\begin{array}{l}1 \\ \beta\end{array}\right\}\left\{\psi^{1}, \psi^{7}, \psi^{12}\right\}\right)=S_{A}$.

Proof. From $\left(I_{14}\right),\left(I_{15}\right),\left(I_{16}\right)$ and by Corollary

4.2. and by the definition of the formula $\stackrel{1}{\beta}$.

Corollary 4.4. $\left\langle R_{0+}^{P}, L_{2}^{r} \cup A_{r}\right\rangle \notin C n s_{A}^{T} \Rightarrow$

$$
\left[\sim \beta \in \operatorname{Cn}\left(R_{0+}^{P}, L_{T}^{1}\right)\right] .
$$

Proof. Let (1) $\left\langle R_{0+}^{P}, L_{2}^{r} \cup A_{r}\right\rangle \notin C n s_{A}^{T}$ and

(2) $\stackrel{0}{\sim} \beta \notin C n\left(R_{0+}^{P}, L_{T}^{1}\right)$. Hence, by Theorem 3.6.

and $\left(I_{16}\right)$, we get that (3) $C n\left(R_{0+}^{P}, L_{T}^{1} \cup\left\{\begin{array}{l}0 \\ \beta\end{array}\right\}\right)=A \neq$ $S_{A}$. Hence, from (1), $\left(I_{14}\right),\left(I_{15}\right),\left(I_{16}\right)$ and by

Corollary 4.3., one can get that (4) $A=S_{A}$, what contradicts (3).

Now,

Lemma 4.3. $\left\langle R_{0+}^{P}, L_{2}^{r} \cup A_{r}\right\rangle \notin C n s_{A}^{T} \Rightarrow$

$$
\left(\forall \alpha \in \bar{S}_{A}-A^{1}\right)\left(\forall \delta \in \bar{S}_{A}-A^{1}\right)
$$

$\left[C n\left(R_{0+}^{P}, L_{1}^{1} \cup{ }_{\psi^{7} o_{0} u_{27} \sim \psi^{1}} L_{2}^{r} \cup N \ddot{\bar{\Psi}}_{3} \cup\{\delta\} \cup\right.\right.$

$$
\left.\left\{\alpha \rightarrow \psi^{7}, \xi\right\}\right)=S_{A} \Rightarrow \operatorname{Cn}\left(R_{0+}^{P}, L_{1}^{1} \cup\right.
$$

$$
\left.\left.\psi^{7} O_{0} u_{27} \sim \psi^{1} L_{2}^{r} \cup N \ddot{\bar{\Psi}}_{3} \cup\left\{\alpha \rightarrow \psi^{7}, \xi\right\}\right)=S_{A}\right] \text {, }
$$

where

$$
\begin{aligned}
& \stackrel{00}{\delta}=\psi^{7} \rightarrow \stackrel{0}{\delta}, \xi=\psi^{7} \rightarrow\left(\psi^{1} \rightarrow \psi^{12}\right), \\
& A^{1}=C n\left(R_{0+}^{P}, L_{1}^{1} \cup_{\psi^{7} O_{0} u_{27} \sim \psi^{1}} L_{2}^{r} \cup N \stackrel{\bar{\Psi}}{3}_{3} \cup\{\xi\}\right) .
\end{aligned}
$$

Proof. Let

(1) $\left\langle R_{0+}^{P}, L_{2}^{r} \cup A_{r}\right\rangle \notin C n s_{A}^{T}$ 
and

(2) $\neg\left(\forall \alpha \in \bar{S}_{A}-A^{1}\right)\left(\forall \delta \in \bar{S}_{A}-A^{1}\right)$

$$
\begin{aligned}
& {\left[C n \left(R_{0+}^{P}, L_{1}^{1} \cup{ }_{\psi^{7} O_{0} u_{27} \sim \psi^{1}} L_{2}^{r} \cup N \ddot{\bar{\Psi}}_{3} \cup\right.\right.} \\
& \left.\left\{\begin{array}{l}
00 \\
\delta
\end{array}\right\} \cup\left\{\alpha \rightarrow \psi^{7}, \xi\right\}\right)=S_{A} \Rightarrow \operatorname{Cn}\left(R_{0+}^{P}, L_{1}^{1} \cup\right. \\
& \left.\left.\psi^{7} O_{0} u_{27} \sim \psi^{1} L_{2}^{r} \cup N \ddot{\bar{\Psi}}_{3} \cup\left\{\alpha \rightarrow \psi^{7}, \xi\right\}\right)=S_{A}\right],
\end{aligned}
$$

where

$$
\begin{aligned}
& \text { (3) } \stackrel{00}{\delta}=\psi^{7} \rightarrow \stackrel{0}{\delta} \text {, } \\
& \text { (4) } \xi=\psi^{7} \rightarrow\left(\psi^{1} \rightarrow \psi^{12}\right) \text {, } \\
& \text { (5) } A^{1}=\operatorname{Cn}\left(R_{0+}^{P}, L_{1}^{1} \cup_{\psi^{7} O_{0} u_{27} \sim \psi^{1}} L_{2}^{r} \cup\right. \\
& \left.N \ddot{\bar{\Psi}}_{3} \cup\{\xi\}\right)
\end{aligned}
$$

From (2) - (5), it follows that

(6) $\left(\exists \alpha^{\prime} \in \bar{S}_{A}-A^{1}\right)\left(\exists{ }^{00} \delta^{\prime} \in \bar{S}_{A}-A^{1}\right)$

$$
\begin{aligned}
& {\left[\operatorname { C n } \left(R_{0+}^{P}, L_{1}^{1} \cup \psi^{7} O_{0} u_{27} \sim \psi^{1} L_{2}^{r} \cup N \ddot{\bar{\Psi}}_{3} \cup\right.\right.} \\
& \left.\left\{\delta^{\prime}\right\} \cup\left\{\alpha^{\prime} \rightarrow \psi^{7}, \xi\right\}\right)=S_{A} \& \operatorname{Cn}\left(R_{0+}^{P}, L_{1}^{1} \cup\right.
\end{aligned}
$$$$
\left.\psi^{7} o_{0} u_{27} \sim \psi^{1} L_{2}^{r} \cup N \ddot{\bar{\Psi}}_{3} \cup\left\{\alpha^{\prime} \rightarrow \psi^{7}, \xi\right\}\right)=
$$$$
\left.A \neq S_{A}\right] \text {, where }
$$

(7) $\stackrel{00}{\delta^{\prime}}=\psi^{7} \rightarrow \stackrel{0}{\delta^{\prime}}$,

(8) $\xi=\psi^{7} \rightarrow\left(\psi^{1} \rightarrow \psi^{12}\right)$,

(9) $A^{1}=\operatorname{Cn}\left(R_{0+}^{P}, L_{1}^{1} \cup_{\psi^{7} O_{0} u_{27} \sim \psi^{1}} L_{2}^{r} \cup\right.$

$$
\left.N \stackrel{\bar{\Psi}}{3}_{3} \cup\{\xi\}\right) .
$$

From (6) - (9), it follows that

$$
\text { (10) } \begin{aligned}
& \left(\exists \alpha^{\prime} \in \bar{S}_{A}-A^{1}\right)\left(\exists \delta^{\prime} \in \bar{S}_{A}-A^{1}\right) \\
& {\left[C n \left(R_{0+}^{P}, L_{1}^{1} \cup_{\psi^{7} O_{0} u_{27} \sim \psi^{1}} L_{2}^{r} \cup N \ddot{\bar{\Psi}}_{3} \cup\right.\right.} \\
& \left.\left.\left\{\alpha^{\prime} \rightarrow \psi^{7}, \xi\right\}\right)=A \& A \neq S_{A} \& \stackrel{00}{\delta}^{\prime} \notin A\right] .
\end{aligned}
$$

From (10) and by Theorem 3.6., one can obtain that

(11) $\left(\exists \alpha^{\prime} \in \bar{S}_{A}-A^{1}\right)\left(\exists \delta^{\prime} \in \bar{S}_{A}-A^{1}\right)$

$$
\begin{aligned}
& {\left[C n \left(R_{0+}^{P}, L_{1}^{1} \cup \psi_{\psi^{7} O_{0} u_{27} \sim \psi^{1}} L_{2}^{r} \cup N \overline{\bar{\Psi}}_{3} \cup\right.\right.} \\
& \left.\left\{\alpha^{\prime} \rightarrow \psi^{7}, \xi\right\}\right)=A \& \operatorname{Cn}\left(R_{0+}^{P}, L_{1}^{1} \cup\right. \\
& \psi^{7} o_{0} u_{27} \sim \psi^{1} L_{2}^{r} \cup N \ddot{\bar{\Psi}}_{3} \cup\left\{\sim^{00} \delta^{\prime}\right\} \cup \\
& \left.\left.\left\{\alpha^{\prime} \rightarrow \psi^{7}\right\} \cup\{\xi\}\right)=A^{*} \& A^{*} \neq S_{A}\right] .
\end{aligned}
$$

From $\left(I_{11}\right),\left(I_{14}\right),\left(I_{16}\right)$, it follows that (12) $\operatorname{Cn}\left(R_{0+}^{P}, L_{T}^{1}\right) \subseteq \operatorname{Cn}\left(R_{0+}^{P}, L_{1}^{1}\right)$. From $(1)-(12),\left(I_{5}\right),\left(I_{6}\right),\left(I_{8}\right),\left(I_{14}\right)-\left(I_{17}\right)$, by Corollary 4.4., it follows that

(13) $\left(\exists \alpha^{\prime} \in \bar{S}_{A}-A^{1}\right)\left(\exists \delta^{\prime} \in \bar{S}_{A}-A^{1}\right)$ $\left[C n\left(R_{0+}^{P}, L_{1}^{1} \cup{ }_{\psi^{7} O_{0} u_{27} \sim \psi^{1}} L_{2}^{r} \cup N \stackrel{\bullet}{\Psi}_{3} \cup\right.\right.$

$$
\begin{aligned}
& \left.\left\{\begin{array}{c}
00 \\
\delta^{\prime}
\end{array}\right\} \cup\left\{\alpha^{\prime} \rightarrow \psi^{7}\right\} \cup\{\xi\}\right)=A^{*} \& \\
& \psi^{7}, \gamma_{4}^{\prime}, \gamma_{2}^{\prime}, O_{0} \rightarrow \gamma_{0}, \psi^{1} \rightarrow \psi^{12}, \gamma_{0}^{\prime}, \\
& O_{0}, u_{27}, \beta, \psi^{1} \rightarrow \beta^{\prime} \sim \beta, \sim \psi^{1} \in A^{*} \& \\
& \left.A^{*} \neq S_{A}\right] .
\end{aligned}
$$

Hence, from $\left(I_{12}\right)$, it follows that

(14) $\left(\exists \alpha^{\prime} \in \bar{S}_{A}-A^{1}\right)\left(\exists \delta^{\prime \prime} \in \bar{S}_{A}-A^{1}\right)$

$\left[C n\left(R_{0+}^{P}, L_{1}^{1} \cup{ }_{\psi^{7} O_{0} u_{27} \sim \psi^{1}} L_{2}^{r} \cup N \ddot{\bar{\Psi}}_{3} \cup\right.\right.$

$$
\left.\left\{\sim \delta^{\prime 0}\right\} \cup\left\{\alpha^{\prime} \rightarrow \psi^{7}\right\} \cup\{\xi\}\right)=A^{*} \&
$$

$\left.L_{2}^{r} \subseteq A^{*} \& A^{*} \neq S_{A}\right]$.

From (13), (14), and $\left(I_{1}\right)$, it follows that

(15) $\left(\exists \alpha^{\prime} \in \bar{S}_{A}-A^{1}\right)\left(\exists \delta^{\prime} \in \bar{S}_{A}-A^{1}\right)$

$\left[C n\left(R_{0+}^{P}, L_{1}^{1} \cup{ }_{\psi^{7} O_{0} u_{27} \sim \psi^{1}} L_{2}^{r} \cup N \stackrel{\bar{\Psi}}{3}_{3} \cup\right.\right.$

$\left.\left\{\sim \delta^{00}\right\} \cup\left\{\alpha^{\prime} \rightarrow \psi^{7}\right\} \cup\{\xi\}\right)=A^{*} \&$ $\left.\psi^{7}, \psi^{7} \rightarrow \psi^{1}, \sim \psi^{1}, \psi^{1} \in A^{*} \& A^{*} \neq S_{A}\right]$. 
From (14) and (15), it follows that

(16) $\left(\exists \alpha^{\prime} \in \bar{S}_{A}-A^{1}\right)\left(\exists \delta^{\prime \prime} \in \bar{S}_{A}-A^{1}\right)$

$\left[\operatorname{Cn}\left(R_{0+}^{P}, L_{1}^{1} \cup \psi_{\psi^{7} O_{0} u_{27} \sim \psi^{1}} L_{2}^{r} \cup N \ddot{\bar{\Psi}}_{3} \cup\right.\right.$

$\left.\left\{\sim^{00} \delta^{\prime}\right\} \cup\left\{\alpha^{\prime} \rightarrow \psi^{7}\right\} \cup\{\xi\}\right)=A^{*} \&$

$\left.A^{*} \neq S_{A} \& A^{*}=S_{A}\right]$.

Contradiction.

Lemma 4.4. $\left\langle R_{0+}^{P}, L_{2}^{r} \cup A_{r}\right\rangle \notin C n s_{A}^{T} \Rightarrow$

$\left(\exists \alpha^{\prime} \in \bar{S}_{A}-A^{1}\right)\left(\forall \stackrel{00}{\delta} \in \bar{S}_{A}-A^{1}\right)\left[\operatorname{Cn}\left(R_{0+}^{P}, L_{1}^{1} \cup\right.\right.$

$\left.\left.\psi^{7} o_{0} u_{27} \sim \psi^{1} L_{2}^{r} \cup N \ddot{\bar{\Psi}}_{3} \cup\left\{\begin{array}{l}00 \\ \delta\end{array}\right\} \cup\left\{\alpha^{\prime} \rightarrow \psi^{7}, \xi\right\}\right)=S_{A}\right]$,

where

$$
\stackrel{00}{\delta}=\psi^{7} \rightarrow \stackrel{0}{\delta}, \xi=\psi^{7} \rightarrow\left(\psi^{1} \rightarrow \psi^{12}\right),
$$

$A^{1}=C n\left(R_{0+}^{P}, L_{1}^{1} \cup{\psi^{7} O_{0} u_{27} \sim \psi^{1}}^{r} \cup N \ddot{\bar{\Psi}}_{3} \cup\{\xi\}\right)$.

Proof. Suppose to the contrary that

(1) $\left\langle R_{0+}^{P}, L_{2}^{r} \cup A_{r}\right\rangle \notin C n s_{A}^{T}$

and

(2) $\neg\left(\exists \alpha^{\prime} \in \bar{S}_{A}-A^{1}\right)\left(\forall \stackrel{00}{\delta} \in \bar{S}_{A}-A^{1}\right)$

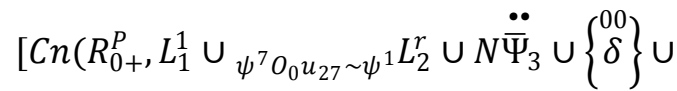

$$
\begin{aligned}
& \left.\left.\left\{\alpha^{\prime} \rightarrow \psi^{7}, \xi\right\}\right)=S_{A}\right],
\end{aligned}
$$

where

(3) $\stackrel{00}{\delta}=\psi^{7} \rightarrow \stackrel{0}{\delta}$,

(4) $\xi=\psi^{7} \rightarrow\left(\psi^{1} \rightarrow \psi^{12}\right)$,

(5) $A^{1}=C n\left(R_{0+}^{P}, L_{1}^{1} \cup{ }_{\psi^{7} O_{0} u_{27} \sim \psi^{1}} L_{2}^{r} \cup N \ddot{\bar{\Psi}}_{3} \cup\right.$

$\{\xi\})$.

Hence,

(6) $\left(\forall \alpha \in \bar{S}_{A}-A^{1}\right)\left(\exists \stackrel{00,}{\delta} \in \bar{S}_{A}-A^{1}\right)$

$$
\begin{aligned}
& {\left[C n \left(R_{0+}^{P}, L_{1}^{1} \cup \psi_{\psi^{7} O_{0} u_{27} \sim \psi^{1}} L_{2}^{r} \cup N \ddot{\bar{\Psi}}_{3} \cup\left\{\delta^{00}\right\} \cup\right.\right.} \\
& \left.\left.\left\{\alpha \rightarrow \psi^{7}, \xi\right\}\right)=A^{+} \neq S_{A}\right],
\end{aligned}
$$

where
(7) $\stackrel{00}{\delta}{ }^{\prime}=\psi^{7} \rightarrow \stackrel{0}{\delta^{\prime}}$,

(8) $\xi=\psi^{7} \rightarrow\left(\psi^{1} \rightarrow \psi^{12}\right)$,

(9) $A^{1}=\operatorname{Cn}\left(R_{0+}^{P}, L_{1}^{1} \cup{ }_{\psi^{7} O_{0} u_{27} \sim \psi^{1}} L_{2}^{r} \cup N \ddot{\bar{\Psi}}_{3} \cup\right.$

$\{\xi\})$.

Next, from $\left(I_{11}\right),\left(I_{14}\right)$ and $\left(I_{16}\right)$, it follows that (10) $\operatorname{Cn}\left(R_{0+}^{P}, L_{T}^{1}\right) \subseteq \operatorname{Cn}\left(R_{0+}^{P}, L_{1}^{1}\right)$.

From (9) and $\left(I_{11}\right)$, it follows that

(11) $\sim \psi^{7} \in A^{1} \Rightarrow\left(\forall \stackrel{0}{\delta} \in \bar{S}_{A}\right)\left[\psi^{7} \rightarrow \stackrel{0}{\delta} \in A^{1}\right]$.

Hence, from (7), it follows that

(12) $\sim \psi^{7} \in A^{1} \Rightarrow \stackrel{00,}{\delta} \in A^{1}$.

Hence, from (6) - (9), it follows that

(13) $\sim \psi^{7} \notin A^{1}$.

From $(6)-(9),\left(I_{5}\right),\left(I_{6}\right)-\left(I_{8}\right),\left(I_{14}\right)-\left(I_{17}\right)$, it follows that

(14) $\left(\forall \alpha \in \bar{S}_{A}-A^{1}\right)\left(\exists \stackrel{00,}{\delta^{\prime}} \in \bar{S}_{A}-A^{1}\right)$

$$
\begin{aligned}
& {\left[C n \left(R_{0+}^{P}, L_{1}^{1} \cup{ }_{\psi^{7} O_{0} u_{27} \sim \psi^{1}} L_{2}^{r} \cup N \stackrel{\bar{\Psi}}{3}_{3} \cup\left\{\delta^{00}\right\} \cup\right.\right.} \\
& \left.\left\{\alpha \rightarrow \psi^{7}, \xi\right\}\right)=A^{+} \& A^{+} \neq S_{A} \& \\
& \gamma_{4}^{\prime}, O_{0} \rightarrow \gamma_{0}, \gamma_{2}^{\prime}, O_{0} \rightarrow\left(u_{27} \rightarrow \stackrel{1}{\beta}\right), O_{0} \rightarrow \stackrel{1}{\beta}, \\
& \left.\gamma_{0}^{\prime} \rightarrow O_{0}, \gamma_{0}^{\prime} \rightarrow \stackrel{1}{\beta}, \beta \in A^{+}\right] .
\end{aligned}
$$

From (1), (6) - (10), (14), $\left(I_{5}\right)-\left(I_{8}\right),\left(I_{11}\right)-\left(I_{17}\right)$, by Corollary 4.4., it follows that

(15) $\left(\forall \alpha \in \bar{S}_{A}-A^{1}\right)\left(\exists \stackrel{00,}{\delta^{\prime}} \in \bar{S}_{A}-A^{1}\right)$

$\left[C n\left(R_{0+}^{P}, L_{1}^{1} \cup \psi_{\psi^{7} O_{0} u_{27} \sim \psi^{1}} L_{2}^{r} \cup N \ddot{\bar{\Psi}}_{3} \cup\left\{\stackrel{00}{\delta}^{\prime}\right\} \cup\right.\right.$ $\left.\left\{\alpha \rightarrow \psi^{7}, \xi\right\}\right)=A^{+} \& A^{+} \neq S_{A} \& \psi^{7} \rightarrow \stackrel{0}{\delta^{\prime}}$,

$\psi^{1} \rightarrow\left(\psi^{7} \rightarrow \psi^{12}\right), \stackrel{1}{\beta}, \psi^{12} \wedge \psi^{7} \wedge \psi^{1} \rightarrow \stackrel{0}{\beta}$,

$\psi^{12} \rightarrow\left(\psi^{7} \rightarrow \sim \psi^{1}\right), \psi^{7} \rightarrow \sim \psi^{1}, \gamma_{4}^{\prime}$,

$O_{0} \rightarrow \gamma_{0}, \gamma_{2}^{\prime}, O_{0} \rightarrow \gamma_{0}^{\prime}, \gamma_{0}^{\prime} \rightarrow O_{0}, \gamma_{0}^{\prime} \rightarrow u_{27}$,

$\psi^{7} \rightarrow\left(\sim \psi^{1} \rightarrow O_{0}\right), \psi^{7} \rightarrow O_{0}, \psi^{7} \rightarrow \gamma_{0}^{\prime}$,

$\psi^{7} \rightarrow u_{27} \in A^{+}$. 
From (15) and $\left(I_{12}\right)$, it follows that

(16) $\left(\forall \alpha \in \bar{S}_{A}-A^{1}\right)\left(\exists \stackrel{00}{\delta^{\prime}} \in \bar{S}_{A}-A^{1}\right)$

$$
\left[\psi^{7} O_{0} \sim \psi^{1} L_{2}^{r} \subseteq A^{+}\right] .
$$

From (15) and (16), it follows that

(17) $\left(\forall \alpha \in \bar{S}_{A}-A^{1}\right)\left(\exists \stackrel{00,}{\delta} \in \bar{S}_{A}-A^{1}\right)\left[O_{6} \in A^{+}\right]$,

where

(18) $O_{6}=O_{0} \rightarrow\left(\psi^{7} \rightarrow \psi^{1}\right)$.

Hence, from (15) and $\left(I_{6}\right)$, it follows that

(19) $\left(\forall \alpha \in \bar{S}_{A}-A^{1}\right)\left(\exists \stackrel{00}{\delta^{\prime}} \in \bar{S}_{A}-A^{1}\right)$

$$
\begin{aligned}
& {\left[C n \left(R_{0+}^{P}, L_{1}^{1} \cup{ }_{\psi^{7} O_{0} u_{27} \sim \psi^{1}} L_{2}^{r} \cup N \ddot{\bar{\Psi}}_{3} \cup\right.\right.} \\
& \left.\left\{\begin{array}{l}
00 \\
\delta^{\prime}
\end{array}\right\} \cup\left\{\alpha \rightarrow \psi^{7}, \xi\right\}\right)=A^{+} \& A^{+} \neq S_{A} \& \\
& \gamma_{0}^{\prime} \rightarrow\left(\psi^{7} \rightarrow \psi^{1}\right), \psi^{7} \rightarrow \psi^{1}, \\
& \left.\psi^{1} \rightarrow \sim \psi^{7}, \sim \psi^{7} \in A^{+}\right] .
\end{aligned}
$$

Hence, from (9), it follows that

$$
\text { (20) } \begin{aligned}
\left(\forall \alpha \in \bar{S}_{A}-A^{1}\right)\left(\exists \delta^{\prime} \in \bar{S}_{A}-A^{1}\right) \\
\\
{\left[C n \left(R_{0+}^{P}, L_{1}^{1} \cup \psi_{\psi^{7} O_{0} u_{27} \sim \psi^{1}} L_{2}^{r} \cup N \stackrel{\bar{\Psi}}{3}_{3} \cup\left\{\delta^{00}\right\} \cup\right.\right.} \\
\left.\left\{\alpha \rightarrow \psi^{7}, \xi\right\}\right)=A^{+} \& A^{+} \neq S_{A} \& \\
\left.A^{+}-A^{1}=\emptyset \& A^{1} \subseteq A^{+}\right] .
\end{aligned}
$$

Hence, from (19), it follows that

(21) $\sim \psi^{7} \in A^{1}$,

what contradicts with (13).

\section{The Main Result}

Theorem 5.1. $\left\langle R_{0+}^{P}, L_{2}^{r} \cup A_{r}\right\rangle \in C n s_{A}^{T}$.

Proof. Let

1) $\left\langle R_{0+}^{P}, L_{2}^{r} \cup A_{r}\right\rangle \notin C n s_{A}^{T}$.

Hence, by Lemma 4.3. and Lemma 4.4., we obtain that

2) $\left(\exists \alpha^{\prime} \in \bar{S}_{A}-A^{1}\right)\left[C n\left(R_{0+}^{P}, L_{1}^{1} \cup\right.\right.$

$$
\left.\left.\psi^{7} O_{0} u_{27} \sim \psi^{1} L_{2}^{r} \cup N \ddot{\bar{\Psi}}_{3} \cup\left\{\alpha^{\prime} \rightarrow \psi^{7}\right\} \cup\{\xi\}\right)=S_{A}\right],
$$

where
3) $A^{1}=\operatorname{Cn}\left(R_{0+}^{P}, L_{1}^{1} \cup{ }_{\psi^{7} O_{0} u_{27} \sim \psi^{1}} L_{2}^{r} \cup N \stackrel{\ddot{\Psi}}{3}_{3} \cup\{\xi\}\right)$.

From 2) and 3), by Theorem 3.5., it follows that

4) $\left(\exists \alpha^{\prime} \in \bar{S}_{A}-A^{1}\right)\left[\sim\left(\alpha^{\prime} \rightarrow \psi^{7}\right) \in A^{1}\right]$, where

5) $A^{1}=C n\left(R_{0+}^{P}, L_{1}^{1} \cup{ }_{\psi^{7} O_{0} u_{27} \sim \psi^{1}} L_{2}^{r} \cup N \ddot{\bar{\Psi}}_{3} \cup\{\xi\}\right)$.

From 4) and 5), it follows that

6) $\left(\exists \alpha^{\prime} \in \bar{S}_{A}\right)\left[\alpha^{\prime} \notin A^{1} \& \alpha^{\prime} \in A^{1}\right]$,

where

7) $A^{1}=C n\left(R_{0+}^{P}, L_{1}^{1} \cup{ }_{\psi^{7} O_{0} u_{27} \sim \psi^{1}} L_{2}^{r} \cup N \ddot{\bar{\Psi}}_{3} \cup\{\xi\}\right)$.

Contradiction

Theorem 5.2. $\left\langle R_{0+}^{P}, L_{2}^{r^{\prime}} \cup A_{r}^{\prime}\right\rangle \in C n s_{A}^{T}$.

Proof. The proof of this Theorem, is analogical to the proof of Theorem 5.1.

\section{References}

[1] Z. Adamowicz. Diagonal reasonings in mathematical logic, Banach Center Publications, 34:9 - 18 (1995).

[2] D. W. Barnes and G. P. Monro. A simple model for a weak system of arithmetic, Bulletin of Australian Mathematical Society, 11:321 - 323 (1974).

[3] D. Batens. The Consistency of Peano Arithmetic. A Defeasible Perspective, In: P. Allo and B. van Kerkhove (Eds.), Modestly Radical or Radically Modest. Fertschrift for Jean Paul Van Bendegem on the Occasion of his 60th Birthday, Tributes24:11 - 39, College Publications, London 2014.

[4] L. D. Beklemishev. Gödel Incompleteness Theorems and the Limits of Their Applicability. I. (translated by A.

Shtern)Russian Mathematical Surveys, 65:857 - 899 (2010); Uspekhi Matematicheskikh Nauk, 65:61 - 106 (2010).

[5] G. Boolos. The Unprovability of Consistency, Cambridge University Press, 2009.

[6] M. Borga and D. Palladino. Logic and foundations of mathematics in Peano's school, Modern Logic, 3:18 - 44 (1992).

[7] B. Buldt. The Scope of Gödel's First Incompleteness Theorem, Logica Univeralis, 8:499 - 552 (2014).

[8] P. Clote and K. McAloon. Two Further Combinatorial Theorems Equivalent to th 1-Consistency of Peano Arithmetic, The Journal of Symbolic Logic, 48:1090 1104 (1983).

[9] J. N. Crossley. What Is a Proof?, In: J. van Benthem, A. Gupta and R. Parikh (Eds.), Proof, Computation and 
Agency. Logic at the Crossroads, pp. 35 - 52, Synthese Library vol. 352, Springer Science \& Business Media B.V., 2011.

[10] C. Dimitracopoulos. Review: Richard Kaye, Models of Peano Arithmetic, The Journal of Symbolic Logic, 58:357 - 358 (1993).

[11] Yu. L. Ershov and E. A. Palyutin. Mathematical Logic, (translated by V. Shokurov), Mir Publishers, Moscow, 1984.

[12] S. Feferman. Arithmetization of Metamathematics in a General Setting, Fundamenta Mathematicae, 49:35 - 92 (1960).

[13] A. M. Fernandes and F. Ferreira. Groundwork for Weak Analysis, The Journal of Symbolic Logic, 67:557 - 578 (2002).

[14] F. Ferreira and G. Ferreira. Interpretability in Robinson's Q, The Bulletin of Symbolic Logic, 19:289 - 317 (2013).

[15] Y. Gauthier. Kronecker in Contemporary Mathematics. General Arithmetic as a Foundantional Programme, Reports on Mathematical Logic, 48:37 - 65 (2013).

[16] A. Grzegorczyk. An Outline of Mathematical Logic. Fundamental Results and Notions Explained with All Details, (translated by O. Wojtasiewicz and W. Zawadowski), Synthese Library, Vol. 70, D. REIDEL PUBLISHING COMPANY, Dordrecht-Holland/Boston-USA, PWN, Warszawa, 1974.

[17] P. Hájek. Kurt Gödel, Completeness, Incompleteness, Journal of Physics: Conference Series, 82:012005 - 012008 (2007).

[18] P. Hájek and P. Pudlák. Metamathematics of First-Order Arithmetic, Springer Verlag, Berlin Heidelberg 1998.

[19] J. Hintikka. Language, Truth and Logic in Mathematics, Springer Science \& Business Media, 1998.

[20] A. Indrzejczak. Jaskowski and Gentzen Approaches to Natural Deduction and Related Systems, In: K. Kijania-Placek and J. Woleński (Eds.), The Lvov-Warsaw School and Contemporary Philosophy, pp. 253 - 264, Synthese Library, vol. 273, Springer-Science \& Business Media Dordrecht, 1998.

[21] R. Kaye. Models of Peano Arithmetic, Clarendon Press, Oxford University Press, Oxford and New York 1991.

[22] H. Kotlarski. The Incompleteness Theorems After 70 Years, Annals of Pure and Applied Logic, 126:125 - 138 (2004).

[23] S. Krajewski. Gödel's Theorem and Its Philosophical Interpretations, Wydawnictwo Instytutu Filozofiii Socjologii PAN, Warszawa 2003, (in Polish).

[24] T. Kubiński. A Proof of Consistency of Borkowski's Logical System Containing Peano's Arithmetic, Studia Logica, 14:197 - 225 (1963).

[25] R. Lassaigne and M. de Rougemont. Logic and Complexity. Springer-Verlag, London 2004.
[26] D. Leivant. Strong Normalization for Arithmetic (Variations on a Theme of Prawitz), In: J. Diller and G. H.Müller (Eds.), ISILC - Proof Theory Symposion. Dedicated to Kurt Schütte on the Occasion of His 65th Birthday Proceedings of the International Summer Institute and Logic Colloquium, Kiel 1974. Lecture Notes in Mathematics, Vol. 500, pp. 182 - 197, Springer-Verlag, Berlin Heidelberg New York 1975.

[27] W. Marciszewski. Logic from a Rhetorical Point of View, Walter de Gruyter, Berlin, New York 1994.

[28] V. W. Marek and J. Mycielski. Foundations of Mathematics in the Twentieth Century, The American Mathematical Monthly, 108:449 - 468 (2001); also in: A. Shenitzer and J. Stillwell (Eds.), Mathematical Evolutions, pp. 225 - 246, The Mathematical Association of America 2002.

[29] S. McCall. The Consistency of Arithmetic: And Others Essays, Oxford University Press, 2014.

[30] R. K. Meyer. Relevant Arithmetic (Abstract), Bulletin of the Section of Logic, 5:133 - 135 (1976).

[31] R. Murawski. On the Incompleteness of Arithmetic Once More, In: J. Perzanowski (Ed.), Essays on Philosophy and Logic. Proceedings of The XXXth Conference on the History of Logic, Dedicated to Roman Suszko, Cracow, October 19-21, 1984, pp. 93 - 102, Jagiellonian University Press, Cracow 1987.

[32] R. Murawski. Hilbert's Program: Incompleteness Theorems vs. partial realizations. In: J. Woleński (Ed.), Philosophical Logic in Poland, pp. 103 - 127, Synthese Library, Vol. 228, Springer-Science \& Business Media Dordrecht, 1994.

[33] R. Murawski. The Contribution of Polish Logicians to Recursion Theory, In: K. Kijania-Placek and J. Woleński (Eds.), The Lvov-Warsaw School and Contemporary Philosophy, pp. 265 - 282, Synthese Library, vol. 273, Springer-Science \& Business Media Dordrecht 1998.

[34] R. Murawski. Recursive Functions and Metamathematics. Problems of Completeness and Decidability, Gödel's Theorems., Synthese Library, Vol. 286, Springer Science \& Business Media Dordrecht, 1999.

[35] R. Murawski. On Proofs of The Consistency of Arithmetic, Studies in Logic, Grammar and Rhetoric, 4:41 - 50 (2001).

[36] E. Nelson. Predicative Arithmetic, Princeton University Press, 1986.

[37] A. Olszewski. Church's Thesis. The HistoricoPhilosophical Context, Monografie Centrum Kopernika, Towarzystwo Autorów i Wydawców Prac Naukowych UNIVERSITAS, Krakow 2009, (in Polish).

[38] M. F. Pallares. Extending the First Gentzen's Consistency Proof to the Intuitionistic Case, Logic Journal of the $I G P L, 12: 549-560$ (2004). 
[39] J. Perzanowski. Parainconsistency, or inconsistency tamed, investigated and exploited, Logic and Logical Philosophy, 9:5 - 24 (2001).

[40] A. Pillay. Models of Peano Arithmetic (a survey of basic results). In: C. Berline, K. McAloon and J.-P. Ressayre (Eds.), Model Theory and Arithmetic (Paris, France 1979/80). Lecture Notes in Mathematics, No. 890, pp. 1 12, Springer-Verlag Berlin Heidelberg, 1981.

[41] J. von Plato. Gentzen's Original Proof of the Consistency of Arithmetic Revisited. In: G. Primiero and S. Rahman (Eds.) Acts of Knowledge-History, Philosophy and Logic, pp. 151 - 171, College Publications, 2009.

[42] K. Podnieks. Fourteen Arguments in Favour of a Formalist Philosophy of Real Mathematics, Baltic Journal of Modern Computing, 3:1 - 15 (2015).

[43] J. Pogonowski. Remarks on Intended Models of Mathematical Theories, Lingua Posnaniensis, 53:83100 (2011).

[44] W. A. Pogorzelski. The Classical Propositional Calculus. PWN, Warszawa, 1975, (in Polish).

[45] W. A. Pogorzelski. The Classical Calculus of Quantifiers. PWN, Warszawa, 1981, (in Polish).

[46] W. A. Pogorzelski and S. J. Surma. Reviews, The Journal of Symbolic Logic, 34:99 - 106 (1969).

[47] W. Pohlers. Proof Theory: The First Step into Impredicativity, Universitext, Springer-Verlag Berlin Heidelberg 2009.

[48] B. Poizat. A Course in Model Theory, Springer Science \& Business Media New York 2000.

[49] G. Priest. Inconsistent Arithmetics: Issues Technical and Philosophical, In: V. F. Hendricks and J. Malinowski (Eds.), Trends in Logic: 50 Years of Studia Logica, Trends in Logic, 21:273 - 299 (2003), Springer Science \& Business Media Dordrecht, 2003.

[50] H. Rasiowa. Introduction to Modern Mathematics, (translated by O. Wojtasiewicz), North-Holland Publishing Company Amsterdam, PWN, Warszawa 1973.

[51] W. Rautenberg. A Consise Introduction to Mathematical Logic, Springer Science \& Business Media, 2006.

[52] S. Salehi. Herbrand Consistency of Some Arithmetical Theories, The Journal of Symbolic Logic, 77:807 - 827 (2012); arXiv:1005.2654.

[53] Ch. Sayward. Remarks on Peano Arithmetic, Russell: the Journal of Bertrand Russell Studies, 20:27 - 32 (2000).

[54] Th. Skolem. Peano's Axioms and Models of Arithmetic, Studies in Logic and the Foundations of Mathematics, 16:1 - 14 (1955) - in: L. E. J. Brouwer, E. W. Beth and A. Heyting (Eds.), Mathematical Interpretation of Formal Systems, pp.1 - 14, North-Holland, Amsterdam London 1971.

[55] P. Smith. An Introduction to Gödel's Theorems, Second Edition, Cambridge University Press, 2014.
[56] C. Smorynski. The Incompleteness Theorems, Studies inLogic and the Foundations of Mathematics, 90:821 865(1977) - in: J. Barwise (Ed.), Handbook of Mathematical Logic, pp. 821 - 865, North-Holland, Amsterdam, 1977.

[57] R. M. Smullyan. Gödel's Incompleteness Theorems, Oxford University Press, 1992.

[58] S. M. Srivastava. A Course on Mathematical Logic, Universitext, Springer Science \& Business Media, LLC 2008.

[59] T. J. Stępień and Ł. T. Stępień. On consistency of Peano's Arithmetic System, a talk delivered at "Logic Colloquium 2009” (July 31 - August 5, 2009, Sofia, Bulgaria) - the slides with this talk, have been placed on the website http://lc2009.fmi.uni-sofia.bg/ contributedslides/Stepien,\%20Stepien.pdf .

[60] T. J. Stępień and Ł. T. Stępień. On the consistency of Peano's Arithmetic System., The Bulletin of Symbolic Logic, 16:132 (2010).

[61] T. J. Stepień and L. T. Stepień. Consistency of Peano's Arithmetic System and Structural Incompleteness of Peano's Arithmetic System, in: IECMSA - 2013, August $26-29,2013$, Sarajevo/Bosnia and Herzegovina, $2^{\text {nd }}$ INTERNATIONAL EURASIAN CONFERENCE ON MATHEMATICAL SCIENCES AND APPLICATIONS. ABSTRACT BOOK. PROCEEDING BOOK.

[62] V. Švejdar. Weak Theories and Essential Incompleteness, In: M. Peliš (Ed.), The Logica Yearbook 2007:

Proceedings of the Logica 07 International Conference, pp. 213 - 224, Philosophia Praha 2008.

[63] W. W. Tait. Gödel's Reformulation of Gentzen's First Consistency Proof For Arithmetic: The No-Counterexample Interpretation, The Bulletin of Symbolic Logic, 11:225 - 238 (2005).

[64] J. Tappenden. Geometry and Generality in Frege's Philosophy of Arithmetic, Synthese, 102:319 - 361 (1995).

[65] A. Tarski, A. Mostowski and R. M. Robinson.Undecidable Theories, North-Holland Publishing Company Amsterdam 1971.

[66] N. Tennant. Deflationism and the Gödel Phenomena, Mind, 111:551 - 582 (2002).

[67] K. Trzęsicki. Philosophy of mathematics and computer science, In: D. Surowik (Ed.), Logic, Philosophy and Computer Science. Series: Studies in Logic, Grammar and Rhetoric, 22:47 - 80 (2010).

[68] M. Tsuji, N. C. A. Da Costa and F. A. Doria. The Incompleteness of Theories of Games, Journal of Philosophical Logic, 27:553 - 568 (1998).

[69] A. Urquhart. Emil Post, In: D. M. Gabbay and J. Woods (Eds.), Handbook of the History of Logic, vol. 5, 429 478 (2008). 
[70] A. Visser. The Second Incompleteness Theorem: Reflections and Ruminations. In: L. Horsten and P. Welch (Eds.), Gödel's Disjunction. The scope and limits of mathematical knowledge, Oxford University Press 2016.

[71] S. Walsh. Logicism, Interpretability and Knowledge of Arithmetic, The Review of Symbolic Logic, 7:84 - 119 (2014).

[72] H. Wang. The axiomatization of arithmetic, The Journal of Symbolic Logic, 22:145 - 158 (1957).

[73] A. Weiermann. Phase Transitions for Gödel incompleteness, Annals of Pure and Applied Logic, 157:281 - 286 (2009).

[74] P. D. Welch. Turing's Mathematical Work. In: R. Latała, A. Ruciński, P. Strzelecki, J. Świątkowski, D. Wrzosek and P. Zakrzewski (Eds.), European Congress of Mathematics: Krakow, 2 - 7 July, 2012, pp. 763 - 777, European Mathematical Society, Zürich, 2013.

[75] H. Weyl. Philosophy of Mathematics and Natural Science, (translated by O. Helmer, with a new introduction by F. Wilczek), Princeton University Press, 2009.
[76] R. Wójcicki. Theories, Theoretical Models, Truth. Part II: Tarski's Theory of Truth and Its Relevance for the Theory of Science, Foundations of Science, 4:471 - 516 (1995/96).

[77] A. Wroński. The Distance Function in Commutative $\ell$-semigroups and the Equivalence in Lukasiewicz Logic, Studia Logica, 77:241 - 253 (2004) (H. Ono and T.

Kowalski - Eds., Special Issue Algebra and Substructural Logics Take Two - Part 2).

[78] L. Wroński. Gödel's Theorems - the Proofs. Is the Arithmetic Able to Prove Its Own Consistency ?, Semina Scientarum, 3:71 - 79 (2004), (in Polish).

[79] R. Zach. Hilbert's Program Then and Now, Philosophy of Logic, 5:411 - 447 (2006); arXiv:math/0508572.

[80] W.-R. Zhang and K. E. Peace. Causality Is Logically Definable - Toward an Equilibrium-Based Computing Paradigm of Quantum Agents and Quantum Intelligence (QAQI) (Survey and Research), Journal of Quantum Information Science, 4:227 - 268 (2014). 